\title{
Correction to: "The way she smiles brightens me up": Highlights of parenting an infant in a large Nationally diverse cohort
}

\author{
Maria T. Corkin ${ }^{1}$ D $\cdot$ E. Dando ${ }^{1} \cdot$ E. R. Peterson ${ }^{1} \cdot$ Natalija Andrejic $^{2} \cdot$ Karen E. Waldie $^{1} \cdot$ Elaine Reese $^{3} \cdot$ \\ Susan M. B. Morton ${ }^{4,5}$ \\ Published online: 4 December 2018 \\ (C) Springer Science+Business Media, LLC, part of Springer Nature 2018
}

\section{Correction to: Current Psychology https://doi.org/10.1007/s12144-018-0014-5}

The original version of this article unfortunately contained mistakes.

The likelihood ratios for the regression tables were incorrect and a rounding error had been applied to the $\mathrm{p}$ values, which we have corrected in the revised tables.

Please note this oversight does not change our findings except in one case, i.e. the finding for ethnicity for the Child Health and Development highlight.

We have provided alterations for the text to reflect the change in this finding. Two paragraphs needed to be amended - the first in the results section and the second in the Discussion.

The online version of the original article can be found at https://doi.org/ 10.1007/s12144-018-0014-5

Maria T. Corkin

mcor009@aucklanduni.ac.nz

1 School of Psychology, University of Auckland, Auckland, New Zealand

2 School of Management, Victoria University of Wellington, Wellington, New Zealand

3 Department of Psychology, University of Otago, Dunedin, New Zealand

4 Centre for Longitudinal Research, University of Auckland, Auckland, New Zealand

5 School of Population Health, University of Auckland, Auckland, New Zealand
The authors apologize for any inconvenience this may have caused.

Amended paragraph 1:

Child Health and Development 'Child health and development' was the second most prevalent highlight, reported by $32 \%$ of mothers in our study $(\mathrm{N}=2051)$. A typical example of a highlight in this category is: "watching her grow and develop - meeting all her milestones". Binomial logistic regression found that the odds of reporting 'Child health and development' highlights were greater for primiparous mothers (OR $=2.09$ ) compared to multiparous mothers. We found that European mothers had greater odds of reporting this highlight compared to Asian mothers $(\mathrm{OR}=2.17)$, MELAA mothers $(\mathrm{OR}=2.04)$ and Pacific mothers $(\mathrm{OR}=1.33)$. Mothers who were in the top three quartiles of external support $(\mathrm{OR}=1.38$; 1.34 and 1.38 respectively) had consistently higher odds of reporting this highlight compared to mothers whose rated their external support in the lowest quartile. Similarly, mothers whose ratings of family support were in the second (OR = 1.29) or upper quartile $(\mathrm{OR}=1.39)$ of family support had greater odds of reporting child and development highlights than those reporting the lowest levels of family support (see Table 7).

Amended paragraph 2:

European mothers were more likely to report the highlight 'Child health and development' compared to mothers of Asian, MELAA or Pacific ethnicity. According to TamisLeMonda et al. (2008), the research suggests that parents from collectivist and individualist cultures prioritise different developmental goals for their children. Individualist cultures, which includes the European culture, tend to value developmental goals that enable autonomy, while collectivist cultures tend to prioritise the development goal of relatedness. Reaching developmental milestones, such as beginning to walk and talk, contribute additively towards autonomy (Tamis-LeMonda 
et al. 2008), and these aspects of a child's development are encompassed in this highlight, e.g., "watching her develope [sic] and become a person rather than a baby". This may go some way towards explaining why European mothers were more likely to identify child health and development high-

Table 6 Binary logistic regression model predicting reporting of the highlight: "Enjoyment of Child" using an Alpha level of .003

\begin{tabular}{llll}
\hline & \multicolumn{2}{c}{ Likelihood Ratio $\left.\mathrm{X}^{2}(60)=156.752, \mathrm{p}<.001\right)$} \\
\cline { 2 - 4 } Variable & Coeff(SE) & OR $(95 \% \mathrm{CI})$ & $p$ value \\
\hline Intercept & & & .007 \\
Ethnicity & & $\mathbf{. 0 0 3}$ \\
European & & & Ref \\
Maori & $\mathbf{0 . 2 8 ( 0 . 0 9 )}$ & $\mathbf{1 . 3 2}(\mathbf{1 . 1 0 - 1 . 5 9 )}$ & $\mathbf{. 0 0 3}$ \\
Pacific Peoples & $0.03(0.10)$ & $1.03(0.84-1.26)$ & .769 \\
Asian & $-0.19(0.09)$ & $0.83(0.69-1.00)$ & .045 \\
MELAA & $0.09(0.21)$ & $1.09(0.72-1.65)$ & .670 \\
NZ/Other & $-0.21(0.26)$ & $0.81(0.48-1.36)$ & .422 \\
Parenting satisfaction & & & $\mathbf{. 0 0 2}$ \\
Low $(M=18.67)$ & & & Ref \\
Medium $(M=21.55)$ & $0.21(0.08)$ & $1.23(1.06-1.44)$ & .008 \\
High $(M=23.58)$ & $\mathbf{0 . 2 8 ( 0 . 0 8 )}$ & $\mathbf{1 . 3 2 ( 1 . 1 3 - 1 . 5 4 )}$ & $\mathbf{. 0 0 1}$ \\
One child only & $\mathbf{0 . 5 4 ( 0 . 0 7 )}$ & $\mathbf{1 . 7 1 ( 1 . 5 0 - 1 . 9 5 )}$ & $<.001$ \\
\hline
\end{tabular}

Alpha adjusted using Benjamini and Hochberg's (1995) method for controlling the false discovery rate. The statistically significant predictor(s) in each model are printed in bold. $\mathrm{R}^{2}=.03$ (Cox \& Snell), .04 (Nagelkerke) MELAA, Middle Eastern, Latin American or African; Coeff, Coefficient; $S E$, Standard Error; OR, Odds Ratio; CI, Confidence Interval; Ref, reference category lights than mothers of Asian, Pacific or MELAA ethnicities, who may endorse more collectivist cultures (Podsiadlowski and Fox 2011).

Amended Tables:

Table 7 Binary logistic regression model predicting reporting of the highlight: "Child's Health and Development" using an Alpha level of .008

\begin{tabular}{|c|c|c|c|}
\hline \multirow[b]{2}{*}{ Variable } & \multicolumn{3}{|c|}{$\left(\right.$ Likelihood Ratio $\left.X^{2}(60)=350.003, p<.001\right)$} \\
\hline & Coeff $(S E)$ & OR $(95 \% \mathrm{CI})$ & $p$ value \\
\hline Intercept & & & $<.001$ \\
\hline Ethnicity & & & $<.001$ \\
\hline European & & & Ref \\
\hline Maori & $-0.27(0.10)$ & $0.76(0.62-0.93)$ & .009 \\
\hline Pacific Peoples & $-0.29(0.11)$ & $0.75(0.60-0.93)$ & .008 \\
\hline Asian & $-0.78(0.11)$ & $0.46(0.37-0.56)$ & $<.001$ \\
\hline MELAA & $-0.71(0.26)$ & $0.49(0.30-0.81)$ & .006 \\
\hline NZ/Other & $-0.43(0.28)$ & $0.65(0.38-1.12)$ & .123 \\
\hline Family support & & & .003 \\
\hline Quartile $1(M=14.28)$ & & & Ref \\
\hline Quartile $2(M=19.50)$ & $0.25(0.09)$ & $1.29(1.09-1.53)$ & .004 \\
\hline Quartile $3(M=23.76)$ & $0.12(0.09)$ & $1.13(0.95-1.34)$ & .179 \\
\hline Quartile $4(M=29.91)$ & $0.33(0.10)$ & $1.39(1.14-1.69)$ & .001 \\
\hline External support & & & .001 \\
\hline Quartile $1(M=12.97)$ & & & Ref \\
\hline Quartile $2(M=17.01)$ & $0.33(0.09)$ & $1.38(1.16-1.65)$ & $<.001$ \\
\hline Quartile $3(M=19.96)$ & $0.29(0.09)$ & 1.34(1.11-1.61) & .002 \\
\hline Quartile $4(M=21.62)$ & $0.32(0.09)$ & $1.38(1.15-1.66)$ & $<.001$ \\
\hline One child only & $0.74(0.07)$ & $2.09(1.83-2.40)$ & $<.001$ \\
\hline
\end{tabular}

Alpha adjusted using Benjamini and Hochberg's (1995) method for controlling the false discovery rate. The statistically significant predictor(s) in each model are printed in bold. $\mathrm{R}^{2}=.07$ (Cox \& Snell), .09 (Nagelkerke) MELAA, Middle Eastern, Latin American or African; Coeff, Coefficient; $S E$, Standard Error; OR, Odds Ratio; $C I$, Confidence Interval; Ref, reference category 
Table 8 Binary logistic regression model predicting reporting of the highlight: "Positive Effects on Family Relationships" using an Alpha level of .005

Table 9 Binary logistic regression model predicting reporting of the highlight: "Characteristics of Child" using an Alpha level of $<.001$

\begin{tabular}{|c|c|c|c|}
\hline \multirow[b]{2}{*}{ Variable } & \multicolumn{3}{|c|}{$\left(\right.$ Likelihood Ratio $\left.X^{2}(60)=764.617, p<.001\right)$} \\
\hline & Coeff $(S E)$ & OR $(95 \% \mathrm{CI})$ & $p$ value \\
\hline Intercept & & & .002 \\
\hline Mother's education level & & & $<.001$ \\
\hline Diploma/Trade cert/NCEA & & & Ref \\
\hline Level $5 / 6$ & & & \\
\hline No secondary school qual & $-0.62(0.18)$ & $0.54(0.38-0.77)$ & .001 \\
\hline Secondary school & $-0.01(0.10)$ & $0.99(0.82-1.20)$ & .950 \\
\hline Bachelor's degree & $0.34(0.10)$ & $1.40(1.16-1.69)$ & .001 \\
\hline Higher degree & $0.29(0.11)$ & $1.34(1.08-1.66)$ & .009 \\
\hline Ethnicity & & & $<.001$ \\
\hline European & & & Ref \\
\hline Maori & $-0.38(0.12)$ & $0.68(0.54-0.87)$ & .002 \\
\hline Pacific Peoples & $-0.44(0.13)$ & $0.65(0.50-0.83)$ & .001 \\
\hline Asian & $-0.28(0.12)$ & $0.75(0.60-0.95)$ & .015 \\
\hline MELAA & $-0.22(0.26)$ & $0.80(0.48-1.34)$ & .399 \\
\hline NZ/Other & $0.60(0.27)$ & $1.83(1.07-3.11)$ & .026 \\
\hline Parenting satisfaction & & & .004 \\
\hline Low $(M=18.67)$ & & & Ref \\
\hline Medium $(M=21.55)$ & $-0.01(0.09)$ & $0.99(0.83-1.18)$ & .924 \\
\hline $\operatorname{High}(M=23.58)$ & $-0.27(0.09)$ & $0.77(0.64-0.92)$ & .005 \\
\hline One child only & $-1.57(0.09)$ & $0.21(0.17-0.25)$ & $<.001$ \\
\hline
\end{tabular}

The statistically significant predictor(s) in each model are printed in bold. $\mathrm{R}^{2}=.14$ (Cox \& Snell), .20 (Nagelkerke). Alpha adjusted using Benjamini and Hochberg's (1995) method for controlling the false discovery rate

MELAA, Middle Eastern, Latin American or African; Coeff, Coefficient; SE, Standard Error; OR, Odds Ratio; CI, Confidence Interval; Ref, reference category

\begin{tabular}{|c|c|c|c|}
\hline \multirow[b]{2}{*}{ Variable } & \multicolumn{3}{|c|}{$\left(\right.$ Likelihood Ratio $\left.X^{2}(60)=192.737, p<.001\right)$} \\
\hline & Coeff $(S E)$ & OR $(95 \% \mathrm{CI})$ & $p$ value \\
\hline Intercept & & & $<.001$ \\
\hline Social expectations & & & $<.001$ \\
\hline Meeting & & & Ref \\
\hline Not meeting/somewhat & $0.14(0.13)$ & $1.16(0.90-1.49)$ & .265 \\
\hline Exceeding & $0.39(0.08)$ & 1.47(1.25-1.74) & $<.001$ \\
\hline One child only & $-0.70(0.09)$ & $0.50(0.42-0.59)$ & $<.001$ \\
\hline
\end{tabular}

Alpha adjusted using Benjamini and Hochberg's (1995) method for controlling the false discovery rate. The statistically significant predictor(s) in each model are printed in bold. $\mathrm{R}^{2}=.04$ (Cox \& Snell), .06 (Nagelkerke) Coeff, Coefficient; SE, Standard Error; OR, Odds Ratio; CI, Confidence Interval; Ref, reference category 
Table 10 Binary logistic regression model predicting reporting of the highlight: "Mother-Child Bonding" using an Alpha level of <.001

\begin{tabular}{llll}
\hline & \multicolumn{2}{l}{$\left(\right.$ Likelihood Ratio $\left.\mathrm{X}^{2}(60)=177.519, \mathrm{p}<.001\right)$} \\
\cline { 2 - 4 } Variable & Coeff $(S E)$ & OR $(95 \% \mathrm{CI})$ & $p$ value \\
\hline Intercept & & $\mathbf{2 . 6 7 ( 2 . 1 1 - 3 . 3 7 )}$ & $<.001$ \\
One child only & $\mathbf{0 . 9 8 ( 0 . 1 2 )}$ & $\mathbf{0 0 1}$ \\
\hline
\end{tabular}

Alpha adjusted using Benjamini and Hochberg's (1995) method for controlling the false discovery rate. The statistically significant predictor(s) in each model are printed in bold. $\mathrm{R}^{2}=.03$ (Cox \& Snell), .08 (Nagelkerke)

Coeff, Coefficient; SE, Standard Error; OR, Odds Ratio; CI, Confidence Interval; Ref, reference category
Table 11 Binary logistic regression model predicting reporting of the highlight: "Identity and Personal Growth" using an Alpha level of $<.001$

\begin{tabular}{llll}
\hline & \multicolumn{2}{l}{$\left(\right.$ Likelihood Ratio $\left.\mathrm{X}^{2}(60)=173.518, \mathrm{p}<.001\right)$} \\
\cline { 2 - 4 } Variable & Coeff $(S E)$ & OR $(95 \% \mathrm{CI})$ & $p$ value \\
\hline Intercept & & $\mathbf{2 . 0 0 1}$ \\
One child only & $\mathbf{0 . 9 6 ( 0 . 1 3 )}$ & $\mathbf{2 . 6 1}(\mathbf{2 . 0 4 - 3 . 3 4})$ & $<.001$ \\
\hline
\end{tabular}

Alpha adjusted using Benjamini and Hochberg's (1995) method for controlling the false discovery rate. The statistically significant predictor(s) in each model are printed in bold. $\mathrm{R}^{2}=.03$ (Cox \& Snell), .08 (Nagelkerke) Coeff, Coefficient; SE, Standard Error; OR, Odds Ratio; CI, Confidence Interval; Ref, reference category

Publisher's Note Springer Nature remains neutral with regard to jurisdictional claims in published maps and institutional affiliations. 